\title{
The old enemy: Obstructive uropathy
}

\author{
Navin Kumar Devaraj* \\ Department of Family Medicine, Universiti Putra Malaysia, Selangor, Malaysia
}

\begin{abstract}
This case reports about Madam RN who presented with severe left loin pain for 2 days and was subsequently found to have obstructive uropathy with acute renal failure. Madam RN was fortunate she was referred early to the urology team which perform life-saving procedures on her.
\end{abstract}

\section{Introduction}

Obstructive uropathy is indeed a dreadful condition to have and it's a urological emergency. There are multiple causes depending on age, gender and co-morbidity of the individual from urinary tract stones, malignancy, benign prostatic hypertrophy, posterior urethral valve, uterine fibroid, trauma and infection, among other [1,2].

The following case will reinforce the need to urgently investigate all cases of obstructive uropathy which are often life-threating. Without urgent action by the medical practitioner, obstructive uropathy can often lead to undesirable outcomes to the unfortunate patient.

\section{Case report}

Madam RN is a 63 years old woman, well previously without any known past medical history. She presented to emergency department a day earlier with the complaint of severe left loin pain for about 10 hours. The pain score was 10/10. She never had previous similar episodes before.

There was no fever or vomiting. Due to chaotic situation at the emergency department that day she was treated symptomatically without any investigations done. She was asked to come to the primary care clinic the next day should the pain persists.

The next day I saw her in my primary care clinic. She complained of being in severe pain for the last 26 hours. She said the analgesics that she took didn't help her much. She complained that she had decreased urine output this past two days. However, there was no dysuria or haematuria.

On general inspection, Madam RN appeared to be in great pain. There was great difficulty in moving her to the bed for examination. Her vital signs were normal except for a pulse rate of 110 beats per minutes. On abdominal examination, there was severe left loin tenderness and the left renal punch was positive.

I immediately did the necessary investigations. The infection screen was normal, but what shocked me was the renal profile reading. The urea and creatinine were markedly raised at $15 \mathrm{mmol} / \mathrm{L}$ and $437 \mathrm{umol} / \mathrm{L}$ giving her an estimated Glomerular Filtration Rate (e-GFR) of only 11.

I made the provisional diagnosis of possible obstructive uropathy. I managed to get an urgent ultrasound kidney, bladder and urethra done which showed left hydro-nephrosis and hydro-ureter secondary to a large renal calculus measuring $1.1 \mathrm{~cm}$.
I referred the case to the urology team which arranged an urgent computed tomography urography which confirmed the above findings. She subsequently underwent surgical removal of the stone and a JJ stent was inserted.

She was covered for pyelonephritis as well and had to undergo a few sessions of haemodialysis. She was discharged after 10 days in the ward. At discharge her creatinine reduced to 170 giving her a e-GFR of 34 .

Since this dreadful episode, she has been on regular follow-up at the urology and primary care clinic for the past 8 months. The JJ tube has been removed successfully and her latest e-GFR has picked up to 53 .

\section{Discussion}

Unfortunately, obstructive uropathy accounts for $2 \%$ for all new cases of end stage renal disease(ESRD) that are beginning treatment with nearly $50 \%$ of this patients below the age of 65 years. Patients may be asymptomatic or presents classically with renal colic pain, urinary symptoms such as decreased frequency, hesitancy, incontinence or urgency, fever in cases of infection, or symptoms of acute or chronic kidney disease [3].

Clinical examination may be normal or reveals severe flank tenderness. Besides the baseline urine and blood tests, diagnostic studies would include either of ultrasonography, intravenous urography (IVU), computed tomography and magnetic resonance imaging. Ultrasonography of kidney, bladder and urethra (US KUB) being easily available, is usually the first line investigation. It is however limited by its inability to provide information on the functional aspect and the completeness of the obstruction and may miss some smaller stones [4].

IVU on the other hand will be able to identify the functional aspect and completeness of the obstruction and accurately describe the site and size of the obstructing stones. However, it has the limitation of

Correspondence to: Navin Kumar Devaraj, Department of Family Medicine, Faculty of Medicine and Health Sciences, University Putra Malaysia, 43400 Serdang, Selangor Darul Ehsan, Malaysia, Tel: +603-89472538, +6013-3105381; Fax: +603-89472328; E-mail: knavin59@yahoo.com

Key words: obstructive uropathy, renal failure, JJ tube

Received: November 06, 2017; Accepted: December 07, 2017; Published: December 11, 2017 
being contraindicated in those with renal failure and contrast allergy and also provides limited or no information about intramural or extramural urinary tract pathology [4].

CT urography has better sensitivity and specificity as compared to IVU but is once again limited by the concerns of radiation exposure just like IVU. It is also a more expensive investigation and provide limited information on ureteric walls and intra-luminal urinary tract pathology. Magnetic resonance urography will be able to overcome all the limitations of the previous imaging techniques, with a much better sensitivity and specificity. However, it is also limited by the availability and cost factor [4].

Management is dependent on the cause ranging from surgical removal of stones through percutaneous nephrolithotomy (PCNL) or ureteroscopy, dissolution of stones with extracorporeal shock wave lithotripsy (ESWL), insertion of JJ stent to maintain the urinary flow from the kidney to the urinary bladder, supportive treatment of renal failure with hydration or dialysis and intravenous antibiotics for infections among other [1]. The most important point is to recognise the need for urgent interventions in suspected cases of obstructive uropathy due to the often-life-threatening nature of the aetiological agent.
In summary, this is a case of acute emergency of obstructive uropathy that presented to the primary care clinic where quick and decisive action was taken. It allowed the recovery of the renal function and removal of the obstructing stone that was causing agonising pain to the unfortunate patient.

\section{Acknowledgement}

The author is very grateful to Madam RN for her consent in publishing this case report.

The author declares no conflict of interest.

\section{References}

1. Sutherland RW (2017) Chapter 46: Obstructive Uropathy.National Kidney Foundation Primer on Kidney Diseases E-Book. 397-404.

2. Sorensen MD, Stoller ML (2012) Nephrology Secrets. Chapter 17: Obstructive Uropathy. USA. Elsevier Inc. 119-122.

3. Zeidel ML (2011) Chapter 125: Obstructive uropathy. In: Goldman L, Schaffer A, editors. Goldman's Cecil Medicine, Expert Consult Premium Edition. 24 ${ }^{\text {th }}$ edition. USA: Elsevier Saunders 776-80

4. Sen KK, Mohan BC, Verma BS (2008) Magnetic resonance urography in obstructive uropathy. MJAFI 64: 145-147.s

Copyright: ( 2017 Devaraj NK. This is an open-access article distributed under the terms of the Creative Commons Attribution License, which permits unrestricted use, distribution, and reproduction in any medium, provided the original author and source are credited. 